\title{
Epithelial-mesenchymal transition in liver fibrosis (Review)
}

\author{
YA-LEI ZHAO, RONG-TAO ZHU and YU-LING SUN
}

\begin{abstract}
Department of Hepatobiliary and Pancreatic Surgery, Institute of Hepatobiliary and Pancreatic Diseases, School of Medicine, First Affiliated Hospital of Zhengzhou University, Zhengzhou 450052, P.R. China
\end{abstract}

Received September 9, 2015; Accepted January 7, 2016

DOI: $10.3892 /$ br.2016.578

\begin{abstract}
Liver fibrosis is the result of a sustained wound healing response to sustained chronic liver injury, which includes viral, alcoholic and autoimmune hepatitis. Hepatic regeneration is the dominant outcome of liver damage. The outcomes of successful repair are the replacement of dead epithelial cells with healthy epithelial cells, and reconstruction of the normal hepatic structure and function. Prevention of the development of epithelial-mesenchymal transition (EMT) may control and even reverse liver fibrosis. EMT is a critical process for an epithelial cell to undergo a conversion to a mesenchymal phenotype, and is believed to be an inflammation-induced response, which may have a central role in liver fibrosis. The origin of fibrogenic cells in liver fibrosis remains controversial. Numerous studies have investigated the origin of all fibrogenic cells within the liver and the mechanism of the signaling pathways that lead to the activation of EMT programs during numerous chronic liver diseases. The present study aimed to summarize the evidence to explain the possible role of EMT in liver fibrosis.
\end{abstract}

\section{Contents}

1. Introduction

2. Different cell types in liver fibrosis

3. Basic concept of EMT

4. Type 2 EMTs

5. Biomarkers of EMT

6. EMT in liver fibrosis: TGF- $\beta /$ Smad and non-Smad signaling pathway

7. Controversy

8. Conclusion

Correspondence to: Dr Yu-Ling Sun, Department of Hepatobiliary and Pancreatic Surgery, Institute of Hepatobiliary and Pancreatic Diseases, School of Medicine, First Affiliated Hospital of Zhengzhou University, 1 Jianshe Road, Zhengzhou 450052, P.R. China

E-mail: doctorssh@126.com

Key words: liver fibrosis, hepatic stellate cells, myofibroblasts, fibrogenesis, transforming growth factor- $\beta$

\section{Introduction}

Liver fibrosis is a complex pathophysiological process of numerous chronic liver diseases, which are characterized by the deposition of the extracellular matrix (ECM). Hepatic stellate cells (HSCs), fibroblasts and myofibroblasts participate in the process via different mechanisms. The inevitable consequence of sustainable liver fibrosis is liver cirrhosis and hepatic cancer, and therefore, preventing liver fibrosis is the primary measure. At present, studies focus on the mechanisms that potentially delay the process of liver fibrosis and even reverse it. Accumulating evidence has shown that mesenchymal cells have an important role in hepatic fibrogenesis. The epithelial-mesenchymal transition (EMT) is suggested as one of the important origins of mesenchymal cells.

During the EMT, epithelial cells lose their epithelial characteristics and gradually obtain a mesenchymal phenotype. The source of the mesenchymal cells participating in tissue repair and regeneration remains to be elucidated. Bi et al (1) reported that alendronate sodium significantly arrested the progression of liver fibrosis. Deng et al (2) observed that biliary epithelial cells (BECs) undergoing EMT may contribute to fibrogenesis in biliary atresia by detecting the antigen for cytokeratin-7 (CK-7), heat-shock protein 1 (HSP1), HSP47 and $\alpha$-smooth muscle actin ( $\alpha$-SMA) in liver sections from patients with biliary atresia. This progress involves the switch of cadherin from E-cadherin to N-cadherin, the dissolution of intracellular connections, the upregulation of matrix remodeling factors and the rearrangement of the cellular cytoskeleton.

\section{Different cell types in liver fibrosis}

Liver fibrogenic cells. Liver fibrogenic cells are a heterogenous cell group, which includes the $\alpha-\mathrm{SMA}^{+}$myofibroblasts (MFs). Liver fibrogenic cells may have a major role in liver fibrosis according to recent studies, and the origin of these cells remains to be elucidated. HSCs are considered the major source as they are the main ECM-producing cells in the injured liver. Hepatic MFs may also originate from bone marrow-derived mesenchymal cells and cells from EMT and endothelial-mesenchymal transition (EnMT).

HSCs. Activation of HSCs is a central event in liver fibrosis. Recently, a number of studies have demonstrated that HSCs are derived from mesodermal-derived multipotent 
mesenchymal progenitor cells. HSCs are significant in producing the ECM, particularly collagen type 1, which is regulated by complex stimuli and pathways. Transforming growth factor- $\beta$ (TGF- $\beta$ ) is prominent among these stimuli. TGF- $\beta$ has 3 major isoforms: TGF- $\beta 1$, TGF- $\beta 2$ and TGF- $\beta 3$. Generally, TGF- $\beta 1$ is stored in an inactivated state, and once activated, it will enhance the transcription of the target gene via its receptors to Smad proteins. As it responds to Smad, the further matrix production in HSCs differs between acute and chronic injury (3). In addition to TGF- $\beta$, there are numerous other factors that exhibit profibrogenic effects on HSCs, such as retinoids and angiotensin II (4-6). During liver fibrosis, parenchymal injury and sustained inflammation generate a large panel of signals that induce the activation of quiescent HSCs. HSC activation is associated with the activation of nuclear factor $\kappa \mathrm{B}(\mathrm{NF}-\kappa \mathrm{B})$ and activator protein 1 , which are activated following the stimulation of intracellular signaling cascades. Platelet-derived growth factor has been shown to activate mitogen-activated protein kinase (MAPK) signaling, specifically c-Jun N-terminal protein kinase, extracellular signal-regulated kinase (ERK) and p38, and finally, regulate HSCs proliferation. Following the activation of HSCs, a variety of changes in gene transcription occur. The target genes include, but are not limited to, the following: $\alpha-S M A$, type 1 collagen, $M M P-2, T G F-\beta 1$, TGF- $\beta$ receptors, TIMPs 1 and $2(7,8)$. Persistent activation leads to changes in HSC behavior, such as proliferation, chemotaxis, fibrogenesis and cytokine release, and all these changes are discrete (9). Liver MFs originating from activated HSCs exhibit high proliferative capacity, upregulate the expression of typical mesenchymal cell markers, such as $\alpha$-SMA, type 1 collagen, fibronectin, desmin and vimentin intermediate filaments (10).

Portal fibroblasts (PFs). The PFs are spindle shaped and exhibit biological similarities with activated HSCs; however, they have different genetic profiles and signaling responses $(11,12)$. They are of mesenchymal origins that undergo myofibroblastic differentiation. PFs do not express $\alpha$-SMA, glial fibrillary acidic protein filaments and desmin, cluster of differentiation 146 (CD146) and cellular retinol-binding protein-1 proteins $(13,14)$, nor store retinoids, which is different from HSCs. In response to tissue injury in liver fibrosis, PFs undergo myofibroblastic activation. Proliferation of the MFs originated from PFs primarily occurs in disease associated with ductular reaction and/or cholestasis, in which the initial injury site is the portal area $(15,16)$.

Fibrocytes. Fibrocytes originated from hematopoietic stem cells are capable to differentiate into MFs. Once tissue is damaged, fibrocytes are recruited to the injured organ and secrete growth factors. The migration of fibrocytes is regulated by $\mathrm{C}-\mathrm{C}$ chemokine receptor type 2 (CCR2) and CCR1. Studies have shown that the extent of differentiation into MFs depends on different organs and the type of injury $(17,18)$.

Bone marrow-derived MFs. Certain hepatic MFs can also originate from the bone marrow-derived mesenchymal stem cells (MSCs), which most likely represent a population that is different from hematopoietic-derived fibrocytes $(9,19,20)$.
Other cells. Studies have shown that MFs may also be derived from hepatocytes or cholangiocytes through EMT in the liver (21). Zeisberg et al (22) were the first to report the evidence for hepatocyte EMT in vivo. They demonstrated that in the transgenic mice challenged with CCL4, in which hepatocyte-derived cells are permanently labeled by $\beta$-galactosidase ( $\beta$-gal), $45 \%$ of the cells expressing the fibroblast-specific protein 1 (FSP1) were also positive for $\beta$-gal expression. Furthermore, the CCL4-induced liver fibrosis can be limited by the inhibition of the TGF- $\beta 1$ pathway. As a summary, the results demonstrated that hepatocyte EMT was triggered by TGF- $\beta 1$ and had a role in liver fibrosis. Cholangiocytes symbolize a unique epithelial cell compartment in the diseased liver. The biliary epithelial cells cannot be ruled out of the assumption that liver epithelial cells undergo EMT in liver fibrosis. Upon liver injury, cholangiocytes proliferate and switch from a quiescent to a 'reactive' state. Reactive cholangiocytes are known to express a variety of cytokines and pro-fibrogenic growth factors. They are likely to contribute to fibrosis and inflammation by promoting activation, proliferation and collagen synthesis in the surrounding pro-fibrogenic cells $(23,24)$. However, Omenetti et al $(25)$ showed a complete EMT in an immature cholangiocyte cell line in vitro, suggesting the possibility of direct contribution of cholangiocytes to fibrosis via EMT. In biliary atresia, biliary epithelial cells expressed FSP-1 and vimentin, while hepatocytes did not. Furthermore, the study showed that the expression of mesenchymal markers in biliary epithelial cells was observed in all liver disease with a ductular proliferation component. In mice exposed to common bile duct ligation (BDL), which is an experimental liver fibrosis model that induces strong ductular reaction, biliary epithelial cells underwent EMT, as shown by type I collagen and $\alpha$-SMA expression (26).

\section{Basic concept of EMT}

EMT allows the epithelial cells to lose their polarity, to undergo complex biochemical changes and to assume multiple mesenchymal cell phenotypes, which includes a significantly increased production of ECM components, migratory capacity, invasiveness and elevated resistance to apoptosis. The progress was first described by Hay in 1995 in a chick model of primitive streak formation (27). In 2003, it was agreed at the first meeting of The EMT International Association, that epithelial-mesenchymal transformation and epithelial-mesenchymal transdifferentiation would be termed EMT. In March 2008, EMT was classified into three different subtypes at an EMT meeting at Cold Spring Laboratory based on the different biological contexts in which they occur $(28,29)$. i) The type 1 EMTs are associated with implantation, embryo formation and organ development, neither cause organ fibrosis nor induce invasive phenotype. ii) The type 2 EMTs, in contrast to type 1, are connected to wound healing, tissue regeneration and organ fibrosis, and involve secondary epithelial or endothelial cells transitioning to resident tissue fibroblasts. As is observed during wound healing and tissue regeneration, the type 2 EMTs are positively correlated with inflammation and cease once inflammation is attenuated. iii) The type 3 EMTs are part of the metastatic process, and occur in neoplastic cells that have previously undergone genetic and epigenetic changes. 


\section{Type 2 EMTs}

Type 2 EMTs are associated with organ fibrosis and regeneration occurring in the liver, lung, kidney and intestine. FSP1, $\alpha$-SMA and collagen 1 are the characterized markers of the mesenchymal products generated by the EMTs during the development of organ fibrosis (29-31). The aforementioned markers, along with vimentin, desmin and discoidin domain receptor 2 (DDR2), have been used to distinguish the epithelial cells that are undergoing EMTs in response to ongoing inflammation. With the development of EMTs, these cells continue to exhibit epithelial-specific morphology and molecular markers, such as E-cadherin and cytokeratin, but showed concomitant expression of FSP1 and $\alpha$-SMA. When the epithelial cell markers continue to be expressed, but the mesenchymal cells markers have been already obtained, such cells possibly represent the intermediate stage of EMT, or namely a partial EMT. Eventually these cells ultimately shed all their epithelial markers (including E-cadherin and zonula occludens-1) and acquire a fully fibroblastic phenotype (31) (vimentin, $\alpha$-SMA, FSP1 and $\beta$-catenin), and the cells have undergone complete EMT. In the lineage studies, during the formation of fibroblasts in liver tissues, renal and other organs including lung and heart, this transition was strongly demonstrated (32-34). Studies have demonstrated that endothelial cells can also be devoted to the formation of mesenchymal cells via a process known as EnMT (35). Li et al (36) studied mouse models with cell lineage analysis and demonstrated that mesothelial cells (MCs) expressing Wilms tumor 1 produce HSCs and MFs during liver fibrogenesis. The results suggest that MCs participate in liver injury via differentiation to HSCs and MFs and are able to undergo mesothelial-mesenchymal transition.

An EMT can be identified in rat fetal liver cells in response to growth factors (epithelial growth factor and TGF- $\beta$ ) and dimethyl sulfoxide $(37,38)$. HSCs cultured in vitro were shown to coexpress epithelial and mesenchymal markers, which provided indirect evidence of EMT $(39,40)$. Increasing evidence has shown that TGF- $\beta$ can induce an EMT in mice hepatocytes in vitro. The mechanism demonstrated that TGF- $\beta$ induced EMT via a MAPK-dependent pathway and a Smad2/3-dependent pathway. Studies have shown that hepatic growth factors can decrease the level of TGF- $\beta$, restore E-cadherin, and decrease the amount of active matrix metalloproteinase 9 (MMP-9) (41) potentially. Other studies have demonstrated that the E-cadherin $/ \beta$-catenin signaling axis also has an important role for EMT involving epithelial cells. Bone morphogenetic protein 7 (BMP-7) has been used in the mouse model of liver, kidney and lung fibrosis, and the results demonstrated that BMP-7 functions as an endogenous inhibitor of TGF- $\beta$ induced EMT (31). TGF- $\beta 1$ is recognized as a major cytokine in organ fibrosis and is an inducer of collagen production and HSC proliferation (42).

\section{Biomarkers of EMT}

To demonstrate the EMT, a variety of biomarkers have been used. Among these markers, some are acquired and some are attenuated during the process of transition. The following are a few of the commonly used markers and mechanisms.
A change or switch of E-cadherin during the EMT in tissue fibrosis, cancer and embryonic development is the prototypical epithelial cell marker. During the transition, the expression of E-cadherin is decreased, and in addition, EMT is promoted by the loss of E-cadherin function $(43,44)$. The switches from E-cadherin to N/OB-cadherin have been increasingly used in recent years to monitor the progress of EMT during embryonic development, fibrosis and cancer progression. Integrins are other EMT markers, which in general have limited utility, as various integrins are expressed on mesenchymal and epithelial cells. DDR2 upregulates MMP1 and cell motility upon binding to type 1 or type $\mathrm{X}$ collagen, and is associated with types 2 and 3 EMT. FSP-1 (also known as S100A4 and MTS-1), is a member of the $\mathrm{Ca}^{2+}$-binding S100 proteins. In tissue fibrosis, FSP-1 is expressed by epithelial cells undergoing type 2 EMT transition to mesenchymal cells, and it has been used as a prototypical marker for detecting EMT in fibrosis and cancer. Vimentin, another marker of EMT, is expressed in various cells including fibroblasts and endothelial cells, and it should not be treated as a typical marker of type 2 EMT as adult epithelial cells express vimentin in response to different insults (45). Fibronectin serves as a scaffold for the ECM, which has been used as an indicator of type 1 EMT. The increased fibronectin expression is associated with type 2 and type 3 EMT in vitro.

\section{EMT in liver fibrosis: TGF- $\beta /$ Smad and non-Smad sig- naling pathway}

TGF- $\beta$ is believed to be a potent inducer of EMT and a key mediator of wound healing, fibrosis (46) and cancer. TGF- $\beta 1$ is a well-established cytokine that induces the profibrogenic pathway and fibrosis in liver (47). Furthermore, TGF- $\beta 1$ expression is also associated with morphological alterations, such as EMT in hepatocytes and changes in survival signaling pathways (48). In the TGF- $\beta$ signaling pathway, active TGF- $\beta 1$ ligands initiate signaling by binding to TGF- $\beta$ receptor type I (T $\beta R I)$ and T $\beta R I I$ serine/threonine kinases. T $\beta R I$ phosphorylates Smad2 and Smad3, which form a complex with Smad4 and translocate to the nucleus. Smad proteins convey signals from TGF- $\beta$ to nucleus. Once in the nucleus, the complex of Smads can regulate the transcription of target genes. Activation of several Smad independent pathways have been identified as crucial for EMT induction by TGF- $\beta$, which includes phosphoinositide 3-kinase (PI3K)-Akt (49), focal adhesion kinase (50), p38 MAPK (51), and ERK (52). Recent studies have implicated Krüppel-like factor-8 (53), hyaluronan synthase 2 (54) and microRNA miR-203 (55) as critical regulators of EMT. Kim et al (56) demonstrated that the NF- $\kappa$ B decoy oligodeoxynucleotide inhibited the EMT process in fibrotic liver in vivo. The overexpression of TGF- $\beta 1$ is associated with liver fibrosis in diverse animal models and in patients with chronic liver disease. TGF- $\beta 1$ crucially controls the expression of ECM network components, such as fibrillar collagens and fibronectin, ECM-degrading protease inhibitors plasminogen activator inhibitor-1 and TIMPs and finally regulates ECM deposition. The activity of TGF- $\beta 1$ is strongly induced during chronic liver injury with links between connective tissue growth factor and TGF- $\beta 1$ in the HSC activation process (57), which acquire myofibroblastic features and produce ECM proteins in turn. TGF- $\beta 1$ initiates and maintains 


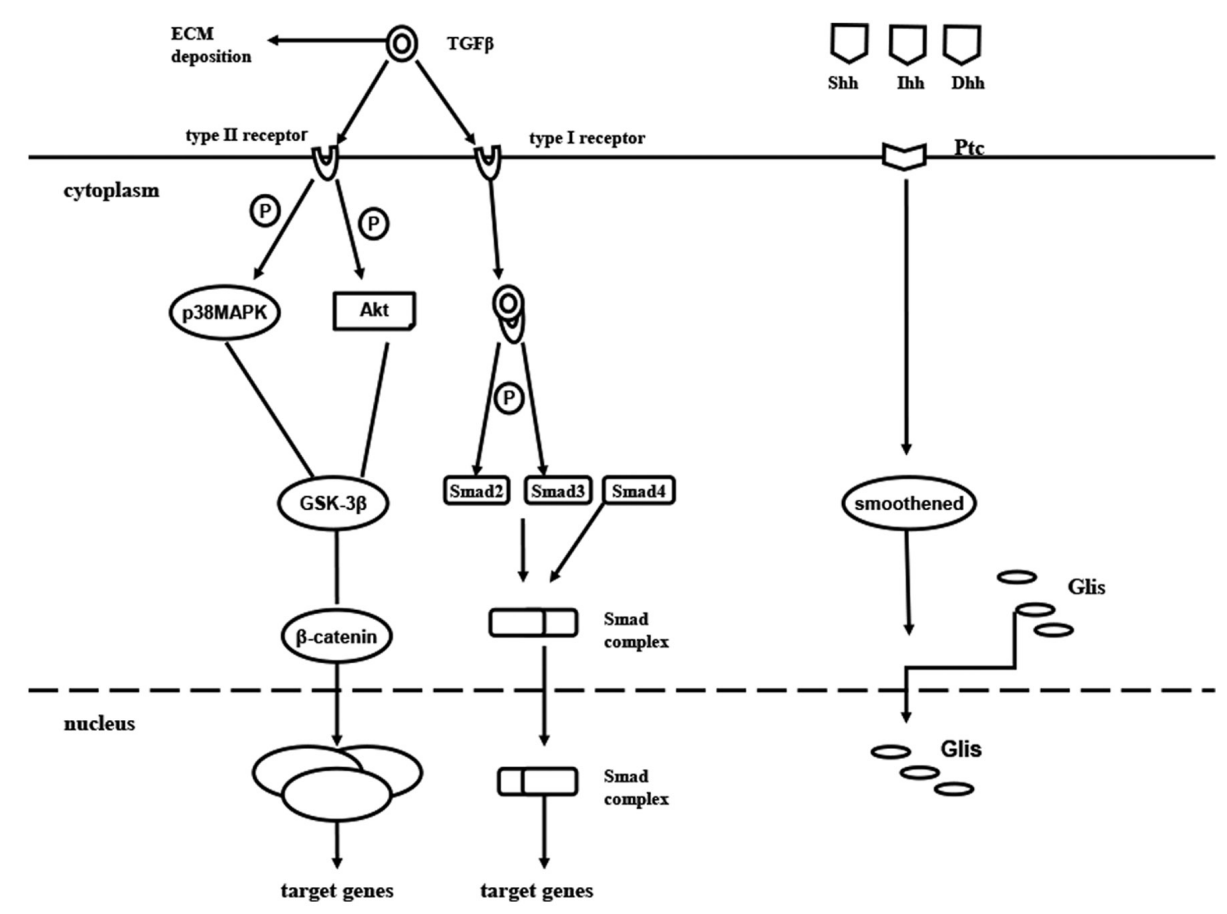

Figure 1. Cellular signaling pathways of EMT in liver fibrosis. Active TGF $\beta 1$ ligands initiate signaling by binding to T $\beta R I$ and T $\beta$ RII serine/threonine kinases. TRRI phosphorylates Smad2 and Smad3, which form a complex with Smad4 and translocate to the nucleus. Smad proteins convey signals from TGF- $\beta$ to the nucleus. Once in the nucleus, the complex of Smad can regulate the transcription of target genes. EMT, epithelial-mesenchymal transition; TGF,

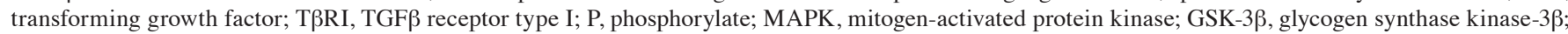
ILK, integrin-linked kinase; TCF/LEF-1 complex, T-cell factor/lymphoid enhancer-binding factor-1 complex; Hh, hedgehog; Shh, Sonic Hh; Ihh, Indian Hh; Dhh, Desert Hh; Ptc, patch; ECM, extracellular matrix; Glis, glioblastoma family.

the EMT in a variety of biological systems by activating major signaling pathways and transcriptional regulators integrated in extensive cellular networks. It has been suggested that the loss of E-cadherin expression in MDCKII cells exposed to TGF- $\beta 1$ occurs through a Smad-independent mechanism, which includes the MAPK and PI3K pathways with expression of Snail. However, a complete transition to the mesenchymal phenotype additionally requires Smad signaling. A previous study reported that TGF- $\beta 1$ participates in the regulation of the Notch signaling pathway (58). A series of the previously mentioned genes and others described to be involved in EMT (includes Notch2 and Snail) were identified as TGF- $\beta 1$ target genes. Park et al (59) reported that geniposide suppresses EMT, which leads to liver fibrosis by inhibiting multiple TGF- $\beta 1$-mediated molecular mediators involved in hepatic injury. Lee et al (60) demonstrated that apamin suppressed the TGF- $\beta 1$-induced hepatocyte EMT in vitro and CCl4-injected fibrosis in vivo.

The hedgehog (Hh) pathway has been identified as an essential morphogene for tissue remodeling in adult tissue. Hh ligands, Sonic Hh (Shh), Indian Hh (Ihh), Desert Hh (Dhh), bind to the patch (Ptc), releasing smoothened (Smo) into the cytosol (61). The aforementioned released Smo promotes the translocation of the cytoplasmic glioblastoma family (Glis: Gli1, Gli2 and Gli3) into the nucleus, which acts as a transcriptional factor, activating Hh signaling (62-64). Evidence has shown that $\mathrm{Hh}$ signaling is activated in damaged liver, where it regulates tissue reconstruction. The level of $\mathrm{Hh}$ expression was suggested to be parallel to the degree of fibrosis (65). Furthermore, Hh signaling has been demonstrated to activate quiescent hepatic stellate cells into MF-HSCs (66) (Fig. 1).

\section{Controversy}

Hepatocyte EMT. EMT was first demonstrated to occur in the fibrosis tissue in the kidney, in vitro (30). Following this, a mice model of renal fibrosis induced by unilateral ureteral obstruction lost the epithelial marker E-cadherin and gained mesenchymal cells markers (such as $\alpha$-SMA). As the origin of fibroblastic cells remains under debate, it is appealing that the liver epithelial cells may have the possibility participate to fibrosis via EMT. Hepatocyte EMT was observed when cells were incubated with TGF- $\beta 1$ (67), which was characterized by a decrease in epithelial marker E-cadherin expression and concomitant acquisition of mesenchymal markers (type I collagen and vimentin). While substantial experimental evidence supports that EMT makes a contribution to embryonic development and tumor metastasis, and renal fibrosis, the role of EMT in liver fibrosis remains under debate. Taura et al (68) bred the triple transgenic mice expressing ROSA26 stop $\beta$-gal, Albumin Cre and collagen $\alpha 1$ (1) green fluorescent protein and induced fibrosis by $\mathrm{CCl} 4$ injections. The study examined the expression of four different mesenchymal markers, which were FSP-1, $\alpha$-SMA, vimentin and desmin. In these studies, the lack of expression of yellow fluorescent protein (YFP) supports the conclusion that EMT does not contribute to fibrosis in these models. Furthermore, the complete absence of its colocalization with YFP suggests that liver epithelial cells do not transition to either mesenchymal cells or MFs via EMT in the mouse models examined (69). 
Cholangiocyte EMT. Cholangiocyte EMT was recently challenged with lineage-tracing methodology. Scholten et al (70) studied several strains expressing Cre under cholangiocyte-, HSC- or FSP-1-specific promoters in two liver fibrosis models (chronic $\mathrm{CCl} 4$ intoxication and common BDL) with Cre-Lox technology for lineage tracing. Following permanent genetic Cre-mediated labeling of cholangiocytes, the fundamental experiment traced the fate of cells expressing K19 in this case. The study concluded that EMT of cholangiocytes identified by genetic labeling does not contribute to liver fibrosis in mice.

\section{Conclusion}

There have been considerable advances in the understanding of the mechanisms of the EMT. The possibility that EMT makes a contribution to liver fibrogenesis reinforced that not only HSCs, but bone marrow-derived cells and circulating fibrocytes, could contribute to this process. The research of EMT in the next few years holds a significant potential as a viable therapeutic target. Future research probes into the molecular similarities and differences among the EMT programs. Furthermore, the identification of the signaling pathways that lead to activation of EMT programs during liver fibrosis is providing novel insights into the plasticity of cellular phenotypes and possible therapeutic interventions.

\section{References}

1. Bi WR, Jin CX, Xu GT and Yang CQ: Effect of alendronate sodium on the expression of mesenchymal-epithelial transition markers in mice with liver fibrosis. Exp Ther Med 5: 247-252, 2013.

2. Deng YH, Pu CL, Li YC, Zhu J, Xiang C, Zhang MM and Guo CB: Analysis of biliary epithelial-mesenchymal transition in portal tract fibrogenesis in biliary atresia. Dig Dis Sci 56: 731-740, 2011.

3. Yoshida $\mathrm{K}$ and Matsuzaki K: Differential regulation of TGF- $\beta /$ Smad signaling in hepatic stellate cells between acute and chronic liver injuries. Front Physiol 3: 53, 2012.

4. Moreno-Alvarez P, Sosa-Garrocho M, Briones-Orta MA, González-Espinosa C, Medina-Tamayo J, Molina-Jijón E, Pedraza-Chaverri J and Macías-Silva M: Angiotensin II increases mRNA levels of all TGF-beta isoforms in quiescent and activated rat hepatic stellate cells. Cell Biol Int 34: 969-978, 2010.

5. Lee YS and Jeong WI: Retinoic acids and hepatic stellate cells in liver disease. J Gastroenterol Hepatol 27 (Suppl 2): S75-S79, 2012.

6. Rippe RA and Brenner DA: From quiescence to activation: Gene regulation in hepatic stellate cells. Gastroenterology 127: 1260-1262, 2004.

7. Mann J, Oakley F, Akiboye F, Elsharkawy A, Thorne AW and Mann DA: Regulation of myofibroblast transdifferentiation by DNA methylation and MeCP2: Implications for wound healing and fibrogenesis. Cell Death Differ 14: 275-285, 2007.

8. Tsukamoto H, She H, Hazra S, Cheng J and Miyahara T: Anti-adipogenic regulation underlies hepatic stellate cell transdifferentiation. J Gastroenterol Hepatol 21 (Suppl 3): S102-S105, 2006.

9. Elpek GÖ: Cellular and molecular mechanisms in the pathogenesis of liver fibrosis: An update. World J Gastroenterol 20 7260-7276, 2014.

10. Fausther M, Lavoie EG and Dranoff JA: Contribution of Myofibroblasts of Different Origins to Liver Fibrosis. Curr Pathobiol Rep 1: 225-230, 2013.

11. Bosselut N, Housset C, Marcelo P, Rey C, Burmester T, Vinh J, Vaubourdolle M, Cadoret A and Baudin B: Distinct proteomic features of two fibrogenic liver cell populations: Hepatic stellate cells and portal myofibroblasts. Proteomics 10: 1017-1028, 2010.

12. Dranoff JA and Wells RG: Portal fibroblasts: Underappreciated mediators of biliary fibrosis. Hepatology 51: 1438-1444, 2010.
13. Uchio K, Tuchweber B, Manabe N, Gabbiani G, Rosenbaum J and Desmoulière A: Cellular retinol-binding protein-1 expression and modulation during in vivo and in vitro myofibroblastic differentiation of rat hepatic stellate cells and portal fibroblasts. Lab Invest 82: 619-628, 2002.

14. Iwaisako K, Brenner DA and Kisseleva T: What's new in liver fibrosis? The origin of myofibroblasts in liver fibrosis. J Gastroenterol Hepatol 27 (Suppl 2): S65-S68, 2012.

15. Tuchweber B, Desmoulière A, Bochaton-Piallat ML, Rubbia-Brandt L and Gabbiani G: Proliferation and phenotypic modulation of portal fibroblasts in the early stages of cholestatic fibrosis in the rat. Lab Invest 74: 265-278, 1996.

16. Kinnman N, Francoz C, Barbu V, Wendum D, Rey C, Hultcrantz R, Poupon R and Housset C: The myofibroblastic conversion of peribiliary fibrogenic cells distinct from hepatic stellate cells is stimulated by platelet-derived growth factor during liver fibrogenesis. Lab Invest 83: 163-173, 2003.

17. Quan TE, Cowper S, Wu SP, Bockenstedt LK and Bucala R: Circulating fibrocytes: Collagen-secreting cells of the peripheral blood. Int J Biochem Cell Biol 36: 598-606, 2004.

18. Strieter RM, Keeley EC, Burdick MD and Mehrad B: The role of circulating mesenchymal progenitor cells, fibrocytes, in promoting pulmonary fibrosis. Trans Am Clin Climatol Assoc 120: 49-59, 2009.

19. Kisseleva T, Uchinami H, Feirt N, Quintana-Bustamante O, Segovia JC, Schwabe RF and Brenner DA: Bone marrow-derived fibrocytes participate in pathogenesis of liver fibrosis. J Hepatol 45: 429-438, 2006.

20. Kisseleva T and Brenner DA: The phenotypic fate and functional role for bone marrow-derived stem cells in liver fibrosis. J Hepatol 56: 965-972, 2012.

21. Forbes SJ and Parola M: Liver fibrogenic cells. Best Pract Res Clin Gastroenterol 25: 207-217, 2011.

22. Zeisberg M, Yang C, Martino M, Duncan MB, Rieder F, Tanjore $\mathrm{H}$ and Kalluri R: Fibroblasts derive from hepatocytes in liver fibrosis via epithelial to mesenchymal transition. J Biol Chem 282: 23337-23347, 2007.

23. Milani S, Herbst H, Schuppan D, Stein H and Surrenti C: Transforming growth factors beta 1 and beta 2 are differentially expressed in fibrotic liver disease. Am J Pathol 139: 1221-1229, 1991.

24. Pinzani M, Milani S, Herbst H, DeFranco R, Grappone C, Gentilini A, Caligiuri A, Pellegrini G, Ngo DV, Romanelli RG and Gentilini P: Expression of platelet-derived growth factor and its receptors in normal human liver and during active hepatic fibrogenesis. Am J Pathol 148: 785-800, 1996.

25. Omenetti A, Porrello A, Jung Y, Yang L, Popov Y, Choi SS, Witek RP, Alpini G, Venter J and Vandongen HM: Hedgehog signaling regulates epithelial-mesenchymal transition during biliary fibrosis in rodents and humans. J Clin Invest 118: 3331-3342, 2008

26. Xia JL, Dai C, Michalopoulos GK and Liu Y: Hepatocyte growth factor attenuates liver fibrosis induced by bile duct ligation. Am J Pathol 168: 1500-1512, 2006.

27. Hay ED: An overview of epithelio-mesenchymal transformation. Acta Anat (Basel) 154: 8-20, 1995.

28. Zeisberg M and Neilson EG: Biomarkers for epithelial-mesenchymal transitions. J Clin Invest 119: 1429-1437, 2009.

29. Strutz F, Okada H, Lo CW, Danoff T, Carone RL, Tomaszewski JE and Neilson EG: Identification and characterization of a fibroblast marker: FSP1. J Cell Biol 130: 393-405, 1995.

30. Okada H, Danoff TM, Kalluri R and Neilson EG: Early role of Fsp1 in epithelial-mesenchymal transformation. Am J Physiol 273: F563-F574, 1997.

31. Zeisberg M, Hanai J, Sugimoto H, Mammoto T, Charytan D, Strutz F and Kalluri R: BMP-7 counteracts TGF-beta1-induced epithelial-to-mesenchymal transition and reverses chronic renal injury. Nat Med 9: 964-968, 2003.

32. Lee HY, Jeon HS, Song EK, Han MK, Park SI, Lee SI, Yun HJ, Kim JR, Kim JS, Lee YC, et al: CD40 ligation of rheumatoid synovial fibroblasts regulates RANKL-mediated osteoclastogenesis: Evidence of NF-kappaB-dependent, CD40-mediated bone destruction in rheumatoid arthritis. Arthritis Rheum 54: 1747-1758, 2006

33. Iwano M, Plieth D, Danoff TM, Xue C, Okada H and Neilson EG: Evidence that fibroblasts derive from epithelium during tissue fibrosis. J Clin Invest 110: 341-350, 2002.

34. Zeisberg EM, Tarnavski O, Zeisberg M, Dorfman AL, McMullen JR, Gustafsson E, Chandraker A, Yuan X, Pu WT, Roberts $\mathrm{AB}$, et al: Endothelial-to-mesenchymal transition contributes to cardiac fibrosis. Nat Med 13: 952-961, 2007. 
35. Potenta S, Zeisberg E and Kalluri R: The role of endothelial-to-mesenchymal transition in cancer progression. Br J Cancer 99: 1375-1379, 2008.

36. Li Y, Wang $\mathrm{J}$ and Asahina $\mathrm{K}$ : Mesothelial cells give rise to hepatic stellate cells and myofibroblasts via mesothelial-mesenchymal transition in liver injury. Proc Natl Acad Sci USA 110 2324-2329, 2013.

37. PaganR,MartínI,LloberaMand VilaróS:Epithelial-mesenchymal transition of cultured rat neonatal hepatocytes is differentially regulated in response to epidermal growth factor and dimethyl sulfoxide. Hepatology 25: 598-606, 1997.

38. Valdés F, Alvarez AM, Locascio A, Vega S, Herrera B, Fernández M, Benito M, Nieto MA and Fabregat I: The epithelial mesenchymal transition confers resistance to the apoptotic effects of transforming growth factor beta in fetal rat hepatocytes. Mol Cancer Res 1: 68-78, 2002.

39. Sicklick JK, Choi SS, Bustamante M, McCall SJ, Pérez EH, Huang J, Li YX, Rojkind M and Diehl AM: Evidence for epithelial-mesenchymal transitions in adult liver cells. Am J Physiol Gastrointest Liver Physiol 291: G575-G583, 2006.

40. Xue ZF, Wu XM and Liu M: Hepatic regeneration and the epithelial to mesenchymal transition. World J Gastroenterol 19: 1380-1386, 2013.

41. Yang J and Liu Y: Blockage of tubular epithelial to myofibroblast transition by hepatocyte growth factor prevents renal interstitial fibrosis. J Am Soc Nephrol 13: 96-107, 2002.

42. Eghbali-Fatourechi G, Sieck GC, Prakash YS, Maercklein P, Gores GJ and Fitzpatrick LA: Type I procollagen production and cell proliferation is mediated by transforming growth factor-beta in a model of hepatic fibrosis. Endocrinology 137: 1894-1903, 1996

43. Hay ED and Zuk A: Transformations between epithelium and mesenchyme: Normal, pathological and experimentally induced. Am J Kidney Dis 26: 678-690, 1995.

44. Huber MA, Kraut N and Beug H: Molecular requirements for epithelial-mesenchymal transition during tumor progression. Curr Opin Cell Biol 17: 548-558, 2005.

45. Witzgall R, Brown D, Schwarz C and Bonventre JV: Localization of proliferating cell nuclear antigen, vimentin, c-Fos and clusterin in the postischemic kidney. Evidence for a heterogenous genetic response among nephron segments and a large pool of mitotically active and dedifferentiated cells. J Clin Invest 93: 2175-2188, 1994.

46. Klass BR, Grobbelaar AO and Rolfe KJ: Transforming growth factor beta1 signalling, wound healing and repair: A multifunctional cytokine with clinical implications for wound repair, a delicate balance. Postgrad Med J 85: 9-14, 2009.

47. Martin M, Lefaix J and Delanian S: TGF-beta1 and radiation fibrosis: A master switch and a specific therapeutic target? Int J Radiat Oncol Biol Phys 47: 277-290, 2000.

48. Del Castillo G, Murillo MM, Alvarez-Barrientos A, Bertran E, Fernández M, Sánchez A and Fabregat I: Autocrine production of TGF-beta confers resistance to apoptosis after an epithelial-mesenchymal transition process in hepatocytes: Role of EGF receptor ligands. Exp Cell Res 312: 2860-2871, 2006.

49. Bakin AV, Tomlinson AK, Bhowmick NA, Moses HL and Arteaga CL: Phosphatidylinositol 3-kinase function is required for transforming growth factor beta-mediated epithelial to mesenchymal transition and cell migration. J Biol Chem 275 36803-36810, 2000

50. Cicchini C, Laudadio I, Citarella F, Corazzari M, Steindler C, Conigliaro A, Fantoni A, Amicone L and Tripodi M: TGFbeta-induced EMT requires focal adhesion kinase (FAK) signaling. Exp Cell Res 314: 143-152, 2008.

51. Bhowmick NA, Zent R, Ghiassi M, McDonnell M and Moses HL: Integrin beta 1 signaling is necessary for transforming growth factor-beta activation of p38MAPK and epithelial plasticity. J Biol Chem 276: 46707-46713, 2001.

52. Xie L, Law BK, Chytil AM, Brown KA, Aakre ME and Moses HL: Activation of the Erk pathway is required for TGF-beta1-induced EMT in vitro. Neoplasia 6: 603-610, 2004.
53. Zhang H, Liu L, Wang Y, Zhao G, Xie R, Liu C, Xiao X, Wu K, Nie Y, Zhang H and Fan D: KLF8 involves in TGF-beta-induced EMT and promotes invasion and migration in gastric cancer cells. J Cancer Res Clin Oncol 139: 1033-1042, 2013.

54. Porsch H, Bernert B, Mehić M, Theocharis AD, Heldin $\mathrm{CH}$ and Heldin P: Efficient TGF $\beta$-induced epithelial-mesenchymal transition depends on hyaluronan synthase HAS2. Oncogene 32: 4355-4365, 2013

55. Ding X, Park SI, McCauley LK and Wang CY: Signaling between transforming growth factor $\beta$ (TGF- $\beta$ ) and transcription factor SNAI2 represses expression of microRNA miR-203 to promote epithelial-mesenchymal transition and tumor metastasis. J Biol Chem 288: 10241-10253, 2013.

56. Kim KH, Lee WR, Kang YN, Chang YC and Park KW: Inhibitory effect of nuclear factor- $\kappa \mathrm{B}$ decoy oligodeoxynucleotide on liver fibrosis through regulation of the epithelial-mesenchymal transition. Hum Gene Ther 25: 721-729, 2014

57. Leask A and Abraham DJ: TGF-beta signaling and the fibrotic response. FASEB J 18: 816-827, 2004.

58. Zavadil J, Cermak L, Soto-Nieves N and Böttinger EP: Integration of TGF-beta/Smad and Jagged1/Notch signalling in epithelial-to-mesenchymal transition. EMBO J 23: 1155-1165, 2004.

59. Park JH, Yoon J, Lee KY and Park B: Effects of geniposide on hepatocytes undergoing epithelial-mesenchymal transition in hepatic fibrosis by targeting TGF//Smad and ERK-MAPK signaling pathways. Biochimie 113: 26-34, 2015.

60. Lee WR, Kim KH, An HJ, Kim JY, Lee SJ, Han SM, Pak SC and Park KK: Apamin inhibits hepatic fibrosis through suppression of transforming growth factor $\beta 1$-induced hepatocyte epithelial-mesenchymal transition. Biochem Biophys Res Commun 450: 195-201, 2014.

61. Wang S, Lee Y, Kim J, Hyun J, Lee K, Kim Y and Jung Y: Potential role of Hedgehog pathway in liver response to radiation. PLoS One 8: e74141,2013.

62. Ingham PW and McMahon AP: Hedgehog signaling in animal development: Paradigms and principles. Genes Dev 15: 3059-3087, 2001

63. van den Brink GR: Hedgehog signaling in development and homeostasis of the gastrointestinal tract. Physiol Rev 87: 1343-1375, 2007.

64. Varjosalo M and Taipale J: Hedgehog: Functions and mechanisms. Genes Dev 22: 2454-2472, 2008.

65. Kahila Bar-Gal G, Kim MJ, Klein A, Shin DH, Oh CS, Kim JW, Kim TH, Kim SB, Grant PR, Pappo O, et al: Tracing hepatitis B virus to the 16th century in a Korean mummy. Hepatology 56: 1671-1680, 2012.

66. Choi SS, Omenetti A, Witek RP, Moylan CA, Syn WK, Jung Y, Yang L, Sudan DL, Sicklick JK, Michelotti GA, et al: Hedgehog pathway activation and epithelial-to-mesenchymal transitions during myofibroblastic transformation of rat hepatic cells in culture and cirrhosis. Am J Physiol Gastrointest Liver Physiol 297: G1093-G1106, 2009.

67. Kaimori A, Potter J, Kaimori JY, Wang C, Mezey E and Koteish A: Transforming growth factor-beta1 induces an epithelial-to-mesenchymal transition state in mouse hepatocytes in vitro. J Biol Chem 282: 22089-22101, 2007.

68. Taura K, Miura K, Iwaisako K, Osterreicher CH, Kodama Y, Penz-Osterreicher $\mathrm{M}$ and Brenner DA: Hepatocytes do not undergo epithelial-mesenchymal transition in liver fibrosis in mice. Hepatology 51: 1027-1036, 2010.

69. Lee SJ, Kim KH and Park KK: Mechanisms of fibrogenesis in liver cirrhosis: The molecular aspects of epithelial-mesenchymal transition. World J Hepatol 6: 207-216, 2014.

70. Scholten D, Osterreicher CH, Scholten A, Iwaisako K, Gu G, Brenner DA and Kisseleva T: Genetic labeling does not detect epithelial-to-mesenchymal transition of cholangiocytes in liver fibrosis in mice. Gastroenterology 139: 987-998, 2010. 